\title{
Spike-timing dependent plasticity and the cognitive map
}

\author{
Daniel Bush ${ }^{1,2, *}$, Andrew Philippides ${ }^{2}$, Phil Husbands $^{2}$ and Michael O'Shea ${ }^{2}$ \\ 1 Department of Physics and Astronomy, University of California Los Angeles, Los Angeles, CA, USA \\ 2 Centre for Computational Neuroscience and Robotics, University of Sussex, Brighton, UK
}

\author{
Edited by: \\ Per Jesper Sjöström, University \\ College London, UK \\ Reviewed by: \\ Niraj S. Desai, The Neurosciences \\ Institute, USA \\ Jack Mellor, Bristol University, UK \\ *Correspondence: \\ Daniel Bush, Department of Physics \\ and Astronomy, University of California \\ Los Angeles, Knudsen Hall 5-170, 475 \\ Portola Plaza, Los Angeles, CA 90095, \\ USA. \\ e-mail:drdanielbush@gmail.com
}

Since the discovery of place cells - single pyramidal neurons that encode spatial location - it has been hypothesized that the hippocampus may act as a cognitive map of known environments. This putative function has been extensively modeled using auto-associative networks, which utilize rate-coded synaptic plasticity rules in order to generate strong bi-directional connections between concurrently active place cells that encode for neighboring place fields. However, empirical studies using hippocampal cultures have demonstrated that the magnitude and direction of changes in synaptic strength can also be dictated by the relative timing of pre-and post-synaptic firing according to a spike-timing dependent plasticity (STDP) rule. Furthermore, electrophysiology studies have identified persistent "theta-coded" temporal correlations in place cell activity in vivo, characterized by phase precession of firing as the corresponding place field is traversed. It is not yet clear if STDP and theta-coded neural dynamics are compatible with cognitive map theory and previous rate-coded models of spatial learning in the hippocampus. Here, we demonstrate that an STDP rule based on empirical data obtained from the hippocampus can mediate rate-coded Hebbian learning when pre- and post-synaptic activity is stochastic and has no persistent sequence bias. We subsequently demonstrate that a spiking recurrent neural network that utilizes this STDP rule, alongside theta-coded neural activity, allows the rapid development of a cognitive map during directed or random exploration of an environment of overlapping place fields. Hence, we establish that STDP and phase precession are compatible with rate-coded models of cognitive map development.

Keywords: STDP, hippocampus, spatial memory, synaptic plasticity, auto-associative network, phase precession, navigation

\section{INTRODUCTION}

The hippocampus and surrounding medial temporal lobe are implicated in declarative memory function in humans and other mammals (Morris, 2007). It has been demonstrated that the firing rate of place cells within the hippocampus encodes for spatial location in the corresponding place field within an environment (O'Keefe and Dostrovsky, 1971; O'Keefe, 2007). It has therefore been suggested that the hippocampus may act as a cognitive map of known locations (O'Keefe and Nadel, 1978; Muller et al., 1991). Behavioral experiments supporting this hypothesis demonstrate that the hippocampus is required for efficient navigation and spatial learning in novel environments (Morris et al., 1982; McNaughton et al., 2006; Morris, 2007).

At the cellular level, processes of learning and memory are believed to be mediated by synaptic plasticity (Hebb, 1949; Martin et al., 2000; Neves et al., 2008). Hippocampal mnemonic function has most frequently been modeled using auto-associative networks - recurrent neural architectures that implement a Hebbian learning rule (Marr, 1971; Hopfield, 1982; Burgess, 2007; Rolls, 2008). The biological correlate of these models is generally taken as the CA3 region of the hippocampus, which exhibits extensive recurrent connectivity and wherein synaptic plasticity can be easily and reliably induced (Rolls and Kesner, 2006; Rolls, 2007).

Initially, empirical studies of activity dependent changes in synaptic strength utilized tetanic stimulation protocols to induce long-term potentiation (LTP) with high frequency stimulation and long-term depression (LTD) with low frequency stimulation (Bliss and Lomo, 1973; Bienenstock et al., 1982; Dudek and Bear, 1992; Malenka and Bear, 2004). Consequently, in models of spatial learning, rate-coded synaptic plasticity rules have been implemented to produce connection weights between place cells that correlate with the relative distance between the corresponding place fields (Muller et al., 1991; Burgess et al., 1994; Burgess, 2007). This approach is supported by evidence for the potentiation of synaptic connections between place cells during exploration of a novel environment (Isaac et al., 2009).

Subsequent research using cultures of hippocampal neurons has demonstrated that bi-directional changes in synaptic strength can also be induced by temporal correlations in low frequency stimulation according to a spike-timing dependent plasticity (STDP) rule (Bi and Poo, 1998; Debanne et al., 1998; Wang et al., 2005). Concurrently, persistent temporal correlations in place cell activity, characterized by the phase precession phenomenon, have been observed during spatial exploration (O'Keefe and Recce, 1993; O'Keefe, 2007; Huxter et al., 2008). Furthermore, the selective elimination of causal temporal correlations in place cell activity prevents the potentiation of interconnecting synapses (Isaac et al., 2009). These findings suggest that rate-based models may not be adequate to replicate the dynamics of hippocampal spatial learning.

Although STDP, combined with the temporally coded patterns of neural activity observed in hippocampus, is ideally suited for route or sequence learning, it has been suggested that the inherent asymmetry of this plasticity rule may not allow the development 
of strong bi-directional connections between place cells that are required by cognitive map theory (Skaggs et al., 1996; Song and Abbott, 2001; Wagatsuma and Yamaguchi, 2007; but see Mongillo et al., 2005; Samura and Hattori, 2005). Computational modeling has also demonstrated that STDP can provide a putative homeostatic function by reducing synaptic currents in response to an increase in input firing rate, and thus provide global stability for local Hebbian learning rules (Song et al., 2000; Song and Abbott, 2001). However, these emergent dynamics directly contradict empirical observations and computational models of rate-coded synaptic plasticity (Bush et al., 2010a).

Here, we present a spiking recurrent neural network model with theta-coded neural dynamics and an STDP rule based on empirical data obtained from the hippocampus in vitro. We demonstrate that this form of STDP is compatible with rate-coded Hebbian learning, producing strong bi-directional connections between neurons that fire stochastically at an elevated rate with no persistent sequence bias. We subsequently demonstrate that this network model can produce a cognitive map of arbitrary one- and two-dimensional environments during shuttle runs and random exploration respectively, such that the magnitude of bidirectional connections between place cells correlates with the relative distance between corresponding place fields. The asymmetry of the plasticity rule dictates that the strength of synaptic connections between place cells are also biased by the frequency and direction in which the corresponding place fields are traversed during exploration. This indicates that STDP can mediate both rate- and temporally coded learning, corresponding to cognitive map formation during open field exploration and sequence learning during route navigation, respectively. However, our results also demonstrate that this form of STDP does not generate any inherent synaptic competition, and some additional mechanism is therefore required to guarantee the long-term stability of network operation.

\section{MATERIALS AND METHODS NEURAL DYNAMICS}

The neural network consists of $N_{p}$ simulated place cells that are fully recurrently connected except for self-connections. Simulated place cells operate according to the Izhikevich (2004) spiking model, which dynamically calculates the membrane potential $(v)$ and a membrane recovery variable $(u)$ based on the values of four dimensionless constants $(a-d)$ and a dimensionless current input $(I)$ according to Eq. 1 . The values used to replicate firing of a standard excitatory neuron are $(a=0.02, b=0.2, c=-65, d=6)$. Under these conditions, simulated neurons fire single spikes at low levels of stimulation, but produce complex spike bursts that are representative of hippocampal pyramidal cells (i.e., several action potentials at a spontaneous rate of $\sim 150 \mathrm{~Hz}$ ) at higher levels of stimulation (Ranck, 1973; Izhikevich, 2004; O’Keefe, 2007).

$$
\begin{aligned}
& v^{\prime}=0.04 v^{2}+5 v+140-u+I \\
& u^{\prime}=a(b v-u) \\
& \text { if } v \geq 30 \text { then }\left\{\begin{array}{l}
v \rightarrow c \\
u \rightarrow u+d
\end{array}\right.
\end{aligned}
$$

Equation 1: The Izhikevich (2004) spiking model.
Each simulated neuron has a randomly chosen axonal delay in the range $(1 \mathrm{~ms}: 5 \mathrm{~ms})$, this being realistic of the CA3 region (Miles, 1990). Previous research has indicated that the scale of axonal delays can have a significant effect on the quantitative, but not qualitative, nature of synaptic dynamics produced by STDP (Bush et al., 2010b). However, it is important to note that the delay between the firing of an action potential and its arrival at post-synaptic targets is unlikely to be uniform for a single neuron in vivo.

\section{THE PLASTICITY MODEL}

Mathematically, with $s=t_{\text {post }}-t_{\text {pre }}$ being the time difference between pre- and post-synaptic spiking, the change in the weight of a synapse $(\Delta w)$ according to a standard implementation of additive STDP can be calculated using Eq. 2. The parameters $A_{+}$and $A_{-}$correspond to the maximum possible change in the synaptic weight per spike pair, while $\tau_{+}$and $\tau_{-}$denote the time constants of exponential decay for potentiation and depression increments respectively. In accordance with experimental observations, coincident pre- and post-synaptic firing elicits maximal depression (Debanne et al., 1998). A nearest-neighbor spike pairing scheme - which dictates that values of $P_{ \pm} \rightarrow A_{ \pm}$ upon afferent or efferent firing - is employed. In all simulations, hard limits are placed on the achievable strength of synapses, such that synaptic weights are maintained continuously in the range $\left(0: w_{\max }\right)$.

Empirical data obtained from the hippocampus suggests that $A_{+}>A_{-}$and $\tau_{+}<\tau_{-}$(Bi and Poo, 1998; Debanne et al., 1998; Wang et al., 2005). Accordingly, we employ values of $\left(A_{+}=0.015\right.$; $\left.A_{-}=-0.012 ; \tau_{+}=20 \mathrm{~ms} ; \tau_{-}=50 \mathrm{~ms}\right)$. Experimental observations in cultures of hippocampal neurons also suggest a non-linear integration of potentiation and depression processes at short temporal offsets (Wang et al., 2005; Pfister and Gerstner, 2006). We therefore utilize an additional potentiation term to replicate this data. Whenever a synaptic connection is depressed, the absolute magnitude of weight decrease is recorded as a parameter $P_{+}$which subsequently decays with a time constant of $\tau_{++}=20 \mathrm{~ms}$. The value of $P_{++}$is then used to supplement any subsequent potentiation of the same synaptic connection (after time $s_{++}=t_{\Delta w+}-t_{\Delta w-}$ ). While this method differs from that employed in the computational model of Pfister and Gerstner (2006), it corresponds to the parameters $A_{3}^{+}$ and $\tau_{x}$ utilized in that study.

It is important to note that the STDP rule utilized here, like the majority of previous computational models, is based primarily on data obtained from cultures of hippocampal pyramidal neurons (Bi and Poo, 1998; Debanne et al., 1998; Wang et al., 2005). More recent empirical studies utilizing acute slices have delineated a more complex relationship between temporal correlations in neural activity and subsequent changes in synaptic strength: primarily, that multiple post-synaptic spikes are required to induce LTP using low frequency temporal correlations (Pike et al., 1999; Meredith et al., 2003; Wittenberg and Wang, 2006; Buchanan and Mellor, 2007; but see Kwag and Paulsen, 2009; Buchanan and Mellor, 2010). In order to retain computational efficiency, we do not aim to explicitly model these findings, but appraise the properties of our STDP rule in light of that data at all relevant junctures. 


$$
\begin{array}{cr}
\Delta w_{+}=F(s)=P_{+}+P_{++} & \text {for } s>0 \\
\Delta w_{-}=F(s)=P_{-} & \text {for } s \leq 0 \\
P_{+}=A_{+}\left(1-\frac{1}{\tau_{+}}\right)^{s} & \\
P_{-}=A_{-}\left(1-\frac{1}{\tau_{-}}\right)^{-S} & \\
P_{++}=\Delta w_{-}\left(1-\frac{1}{\tau_{++}}\right)^{-S_{++}} &
\end{array}
$$

Equation 2: The spike-timing dependent plasticity rule.

\section{SIMULATION DETAILS}

Our initial aim is to characterize the emergent synaptic dynamics produced by the STDP rule described above when neural activity corresponds to a variety of standard stimulation protocols used experimentally to induce LTP and LTD (Bi and Poo, 1998; Wang et al., 2005; Wittenberg and Wang, 2006). Firstly, we examine changes in the strength of bi-directional connections between $N_{p}=2$ simulated neurons when pairs and triplets of pre- and postsynaptic spikes are delivered at low frequency $(1 \mathrm{~Hz})$ for a period of $60 \mathrm{~s}$. These include single pre- and post-synaptic spikes with a temporal offset of $10 \mathrm{~ms}$; a single action potential and a complex burst of two to three spikes with $\sim 5 \mathrm{~ms}$ inter-spike intervals (ISI) offset by $10 \mathrm{~ms}$; and a single action potential with two spikes of differing temporal correlations at various temporal offsets (Figure 1).

Secondly, we examine emergent synaptic dynamics in a small, fully recurrently connected network (excluding self-connections) of $N_{p}=100$ simulated neurons when neural activity corresponds to previous rate-coded auto-associative network models (Marr, 1971; Hopfield, 1982; Burgess, 2007; Rolls, 2008). A subset of simulated place cells $(10 \%)$ fire stochastically at an elevated "foreground" rate $\left(r_{\text {fore }} \approx 20 \mathrm{~Hz}\right)$ while the remainder fire stochastically at a low "background" rate $\left(r_{\text {back }} \approx 0.1 \mathrm{~Hz}\right.$, these values being realistic of CA3) (Frerking et al., 2005). This activity is generated using external excitatory input drawn from a random, uniform distribution in the range $\left(0: I_{\max }\right)$, with $I_{\max }=12$ for foreground neurons and $I_{\max }=4.5$ for background neurons. An examination of the resultant neural dynamics confirms that this form of input produces a Gaussian distribution of ISI (data not shown). In each of these simulations, all synaptic connections in the network are initialized with a weight of $w_{i j}=0.3$ unless specified otherwise.

In further simulations, theta-coded neural dynamics are dictated by a phenomenological phase precession model (O'Keefe and Recce, 1993; O'Keefe, 2007; Huxter et al., 2008). A variable $\theta$, which oscillates sinsuoidally in the range $(0: 1)$ at a rate of $8 \mathrm{~Hz}$, is used to represent theoretical theta frequency oscillations in the local field potential (LFP) that dominate the hippocampal EEG during stereotyped learning behavior (Buzsaki, 2006; O’Keefe, 2007). Inhibitory input to each simulated neuron at each millisecond time step is drawn randomly from a Gaussian distribution with mean $I_{\text {inh }}=-15(1-\theta)$ and standard deviation $\sigma_{\text {inh }}=2$. Neural noise at a rate of $\sim 0.1 \mathrm{~Hz}$ (this being realistic of CA3) is generated in the network by the constant application of excitatory current drawn from a uniform random distribution in the range $\left(0: I_{\text {noise }}\right)$ where $I_{\text {noise }}=0.8$ in all simulations (Frerking et al., 2005). The interplay between afferent inhibitory and excitatory currents means that the majority of firing due to neural noise tends to occur around the peak of the LFP, as defined by the value of $\theta$.

External stimulation of place cells is subsequently provided within different, discrete theta phase windows depending on hypothetical location within (or outside of) the corresponding place field (Figure 2). Circular place fields of $80 \mathrm{~cm}$ diameter are divided into eight equally sized sections of $10 \mathrm{~cm}$, and theta oscillations in the LFP (as defined by the value of $\theta$ ) are similarly divided into "phase windows" of $\pi / 4$ between $\pi / 8$ and $15 \pi / 8$. External input, randomly sampled from a normal distribution with mean $I_{\text {ext }}=0$ and standard deviation $\sigma_{\text {ext }}=30$, is subsequently applied to simulated place cells for the duration of a theta phase window if the hypothetical position lies within the corresponding place field section. This generates a mean in-field firing rate of $\sim 15 \mathrm{~Hz}$, with active place cells tending to fire stochastic bursts at the peak of the LFP (as defined by the value of $\theta$ ) and single spikes on the ascending and descending slope, in accordance with empirical data (O'Keefe

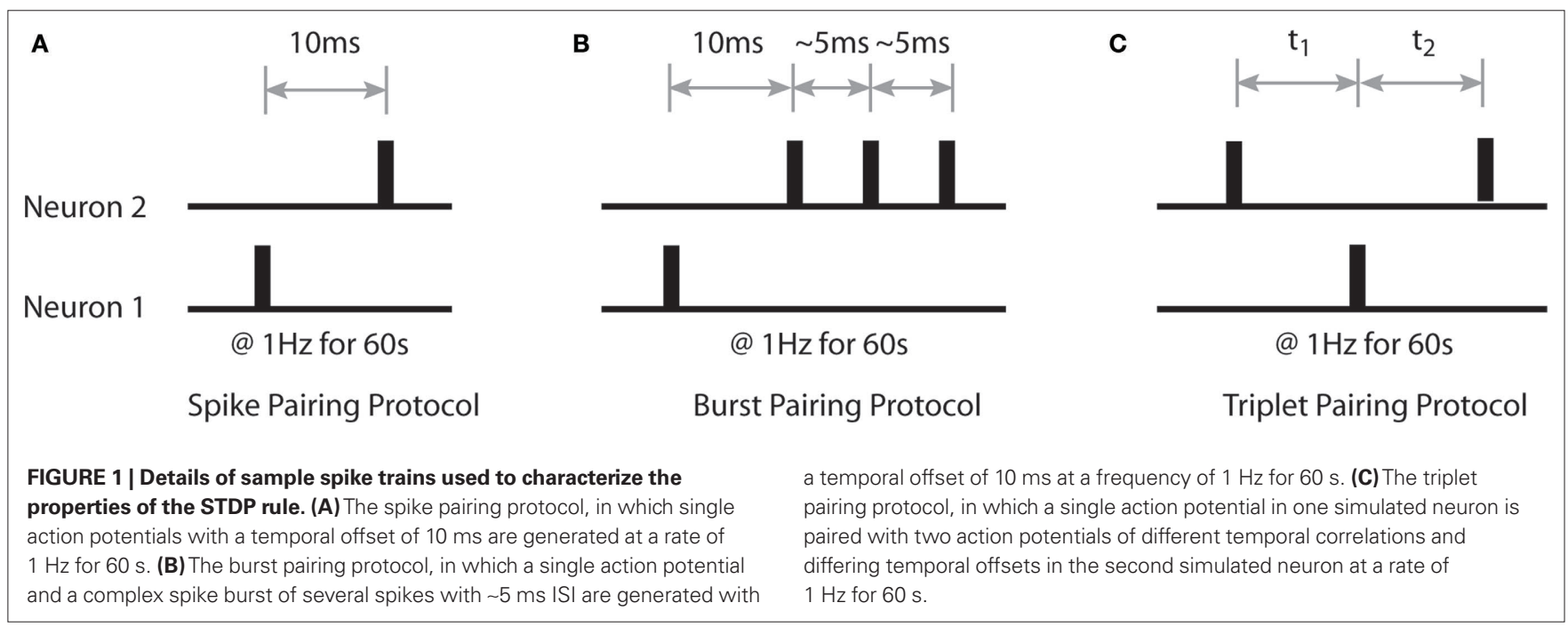




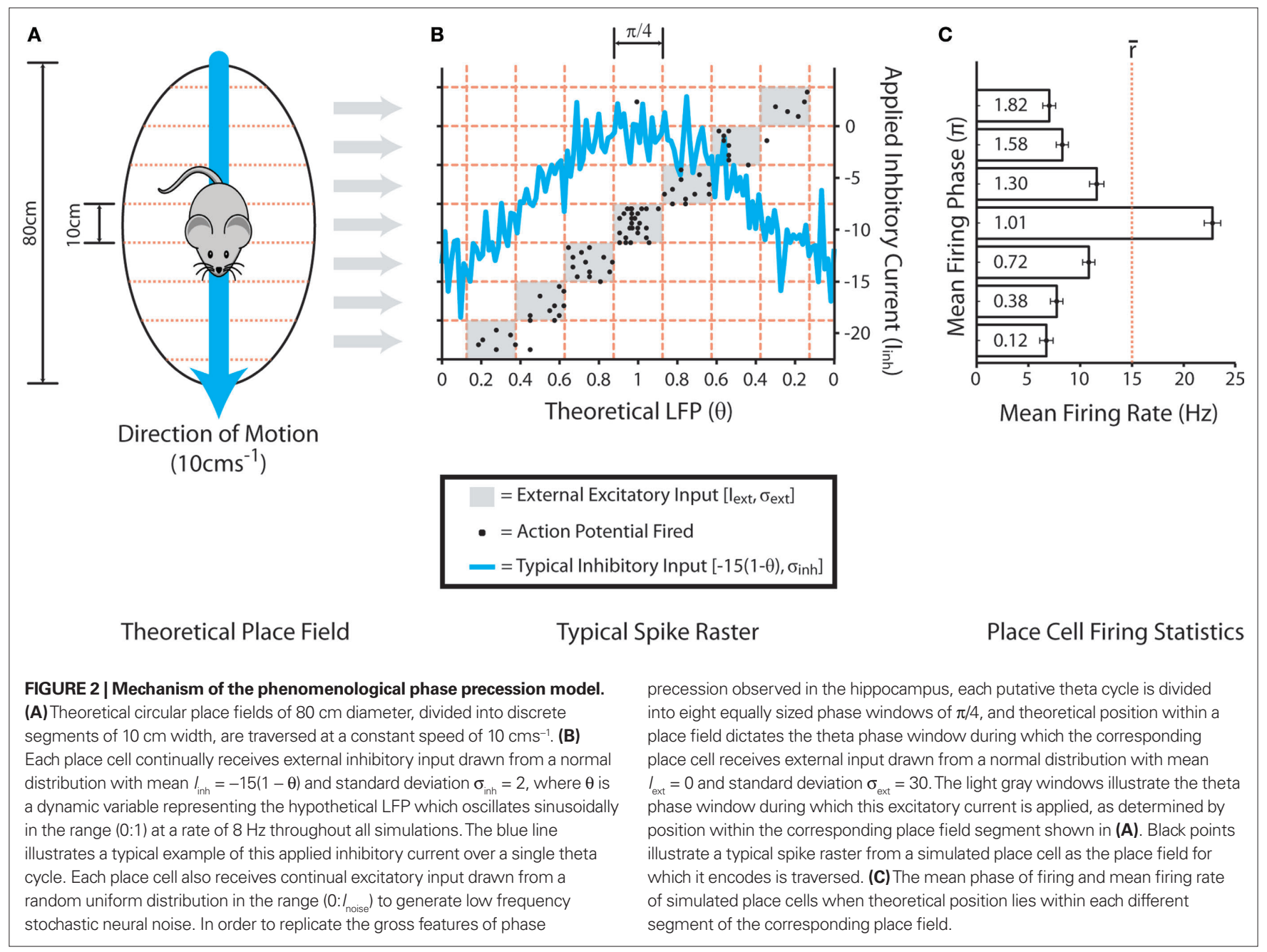

and Recce, 1993; Huxter et al., 2008). It is important to note that the phenomenological phase precession mechanism utilized here, wherein the phase of place cell firing is dictated by the timing of external excitatory input, contrasts with previous computational models which suggest that phase precession may itself be the result of cell assemblies produced by STDP (Jensen and Lisman, 1996; de Almeida et al., 2007). However, there is considerable debate regarding the mechanisms by which theta-coded neural dynamics are produced in vivo, and modeling studies suggest that phase precession in CA3 may largely be inherited from upstream circuits (Baker and Olds, 2007; Maurer and McNaughton, 2007).

Using this phenomenological phase precession model, we first examine changes in the strength of bi-directional connections between $N_{p}=2$ simulated neurons when neural activity corresponds to that observed in hippocampal place cells encoding for overlapping place fields. One simulated place cell consistently fires a complex spike burst (two to three action potentials with $\sim 5 \mathrm{~ms}$ ISI) at the peak of theta (as defined by the value of $\theta$ ) while the second neuron fires stochastically within a different theta phase window for each set of $10 \mathrm{~s}$ simulations performed.

Having established the synaptic dynamics produced by our STDP rule with various sample spike trains, we then examine a fully recurrently connected network of $N_{p}=490$ simulated neurons with theta- coded neural dynamics that correspond to various spatial exploration strategies within a hypothetical square arena. The arena consists of a grid of 49 evenly distributed place fields, each encoded for by 10 place cells, that are offset by $10 \mathrm{~cm}$ and traversed by a hypothetical agent at a constant speed of $10 \mathrm{~cm} \mathrm{~s}^{-1}$. In the first set of "shuttle run" simulations, a randomly selected one-dimensional route of 10 place fields in length is traversed 10 times in alternating directions. Next, in "random exploration" simulations, initial position within the arena is randomly selected, the subsequent direction of movement is chosen randomly at the beginning of each second of simulated time, and thus the agent traverses the arena in an undirected manner for a total period of $490 \mathrm{~s}$. In both cases, these parameter values dictate that there are generally seven different simultaneously active ensembles of 10 place cells, each encoding for a different place field and therefore firing within a different theta phase window. After each $1 \mathrm{~s}$ of simulated time, the mean phase of firing in each place cell ensemble recedes to an earlier theta phase window as the hypothetical agent advances to the next place field segment. In each of these simulations, all synaptic connections in the network are initialized with a weight of $w_{i j}=0.01$ unless specified otherwise.

Experimental data suggests that identical stimulation protocols can induce a differing degree and magnitude of synaptic plasticity within the hippocampus depending on their timing relative to 
ongoing theta oscillations - potentiation generally being incurred at the peak of the LFP, while depression (or depotentiation) is incurred at the trough (Hyman et al., 2003). This is most likely a result of the transient changes in membrane depolarization that proceed over the course of a theta cycle. Here, we perform simulations both with and without plasticity modulation. When dynamic plasticity modulation is implemented, all synaptic weight changes dictated by the STDP rule are scaled by the instantaneous value of $\theta$ according to Eq. 3 .

Fifty independent simulations are performed for each set of experiments described above, and the Mann-Whitney $U$-test is used to assess the significance of differences in resultant synaptic weight distributions.

$\Delta w_{+}=\theta\left(P_{+}+P_{++}\right)$

$\Delta w_{-}=(1-\theta) P_{-}$

Equation 3: Details of the dynamic synaptic plasticity modulation mechanism.

\section{RESULTS}

\section{THE STDP RULE}

In order to appraise the accuracy with which our phenomenological plasticity rule can replicate empirical data obtained from the hippocampus, we first examine the emergent synaptic dynamics generated when neural activity corresponds to several different stimulation protocols commonly used to induce LTP and LTD. Initially, we employ two different spike pairing protocols in which either single pre- and post-synaptic spikes or a single spike and complex burst are paired at short temporal offsets (Figures 1A,B). This generates asymmetric connections that correspond to the "causal" input correlation, with synaptic weights being driven toward either the upper or lower bounds (Figure 3A). These results concur with empirical data obtained using similar stimulation protocols in cultures of hippocampal pyramidal neurons (Bi and Poo, 1998; Debanne et al., 1998; Wang et al., 2005). However, studies using acute hippocampal slices suggest that multiple post-synaptic spikes are required to generate potentiation (Pike et al., 1999; Meredith et al., 2003; Wittenberg and Wang, 2006; Buchanan and Mellor, 2007). Our STDP rule does not replicate this data - although it is important to note that potentiation is more rapid, and thus a greater mean synaptic weight is achieved over the $60 \mathrm{~s}$ stimulation period, when the post-synaptic neuron fires multiple spikes.

Secondly, we employ triplets of pre- and post-synaptic spikes at various temporal offsets (Figure 1C). Under these conditions, the non-linear integration of depression and potentiation processes dictated by the $P_{++}$term generate more complex emergent synaptic dynamics that depend explicitly on the order of spike pairings. When the temporal offset of each spike pairing is equal, then the synaptic connection that experiences a potentiating ("causal") spike pairing after a depressing spike pairing is significantly potentiated (Figure 3B). Conversely, spike pairings in the opposite order (potentiation then depression) generate a much less significant increase in synaptic weight. In the majority of cases - particularly for post-pre-post spike triplets, the magnitude of synaptic changes produced in these simulations approximates that observed in hippocampal cultures when identical stimulation protocols are employed (Wang et al., 2005; Pfister and Gerstner, 2006).
However, asymmetry in the profile of the STDP window employed in this study (i.e., $A_{+}>A_{-}$and $\tau_{+}<\tau_{-}$) dictates that the potentiation and subsequent depression increments produced by pre-post-pre triplets are not equal and do not directly negate. Hence, mild LTP is generated by pre-post-pre triplets with equal temporal offsets (i.e., $t_{1}=t_{2}$ ) and mild LTD is induced when a potentiating spike pairing with a longer temporal offset precedes a depressing increment with a shorter temporal offset (i.e., $w_{21}$ when $\left.t_{1}>t_{2}\right)$. This is in contrast to empirical data, where no significant weight change is observed in either case (Wang et al., 2005; Pfister and Gerstner, 2006). Furthermore, the magnitude of potentiation incurred when a depressing spike pairing with a shorter offset precedes a potentiating increment with a longer temporal offset is much less significant than that observed experimentally. This is surprising, considering the fact that the profile of the STDP window employed in this study is also based directly on empirical data from hippocampal cultures (Bi and Poo, 1998).

Thirdly, we demonstrate that this form of STDP can mediate rate-coded Hebbian learning, in accordance with empirical data regarding observations of LTP induced by tetanic stimulation protocols (Bliss and Lomo, 1973; Malenka and Bear, 2004). When stochastic neural activity at high ("foreground") and low ("background") firing rates is generated in distinct groups of neurons within a small, fully recurrently connected network, those synaptic connections with elevated pre- and post-synaptic firing rate are rapidly, selectively and significantly potentiated (Figures 3C,D). Synaptic connections with solely elevated post-synaptic firing rate undergo modest but continual potentiation, while those with solely elevated pre-synaptic firing rate are significantly depressed. This indicates that the STDP rule reduces the weight of connections that have a negative (i.e., "non-causal") rate correlation. Finally, the strength of synapses with background pre- and post-synaptic firing rate does not change significantly. This is in contrast to the robust LTD usually observed following prolonged low frequency stimulation, and most likely due to the small number of temporally proximate spike pairings that occur at these connections (Dudek and Bear, 1992; Buchanan and Mellor, 2010).

However, over very long periods of simulated time $(-20,000 \mathrm{~s}$ or more) the mean weight of all pre-synaptic connections of neurons firing at an elevated rate saturate at a similarly high value $\left(\sim 0.8 w_{\text {max }}\right.$, data not shown). This indicates that the STDP rule utilized here fails to generate competition between synaptic inputs to a single neuron. This absence of hetero-synaptic competition, and subsequent issues with global network stability, is a common feature of previous rate-coded Hebbian plasticity rules, and require the inclusion of some additional mechanism to ensure stable long-term operation (Desai, 2004).

Finally, we examine the synaptic dynamics generated when neural activity approximates the theta-coded temporal activity correlations observed in place cells encoding for overlapping place fields within the hippocampus (O'Keefe and Recce, 1993; O'Keefe, 2007; Huxter et al., 2008). We consider two bi-directionally connected place cells that fire stochastically at differing mean phase of a putative theta cycle (Figure 4A). Our results demonstrate that asymmetric connections of varying strength are generated when a persistent temporal correlation in preand post-synaptic activity exists, while strong bi-directional 


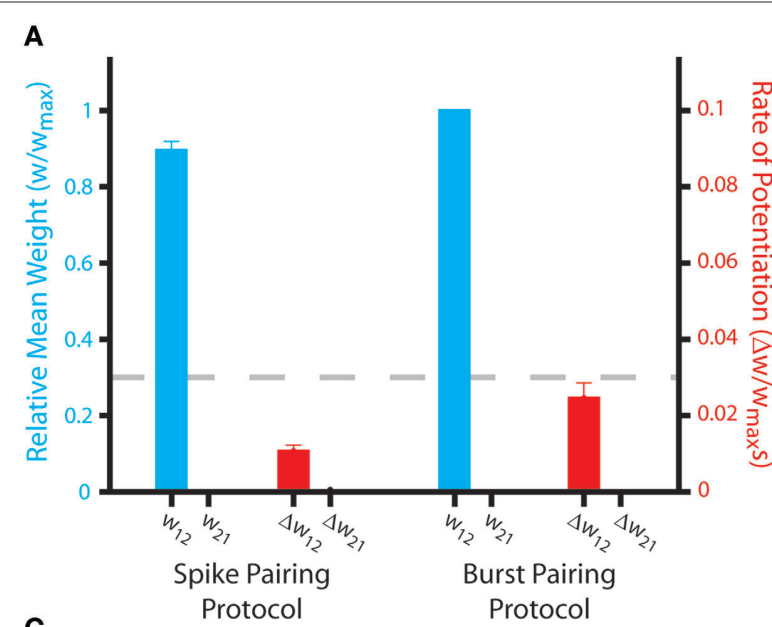

C

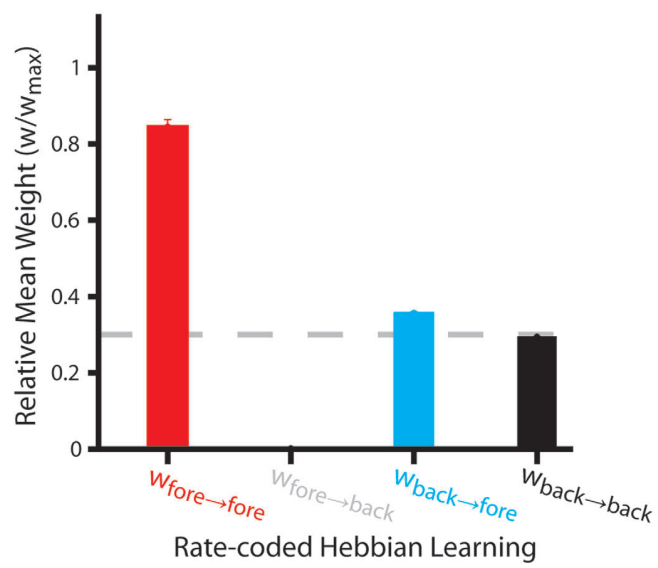

FIGURE 3 | Synaptic weight dynamics produced by the STDP rule with sample rate- and temporally coded spike trains. (A) The final mean weight $\left(W_{i j}\right)$ and rate of weight change per spike pairing $\left(\Delta W_{i j}\right)$ at bi-directional synaptic connections between $N_{p}=2$ simulated neurons over 50 independent

simulations in which single action potentials with an offset of $10 \mathrm{~ms}$ are incurred at a rate of $1 \mathrm{~Hz}$ for $60 \mathrm{~s}$ (the spike pairing protocol, Figure 1A); or a single action potential and complex spike burst with an offset of $10 \mathrm{~ms}$ are incurred at a rate of $1 \mathrm{~Hz}$ for $60 \mathrm{~s}$ (the burst pairing protocol, Figure 1B). The dashed line indicates the initial synaptic weight value of $w_{i j}=0.3$. (B) The final mean weight of bi-directional synaptic connections between $N_{p}=2$ simulated neurons over 50 independent simulations in which triplets of pre- and post-synaptic spikes with differing temporal offsets are incurred at a rate of $1 \mathrm{~Hz}$ for $60 \mathrm{~s}$ (Figure 1C). The dashed line indicates the initial synaptic weight value of $w_{i j}=0.3$. (C) The final

connections are generated when pre- and post-synaptic activity is stochastic but approximately synchronous with no persistent sequence bias (Figures 4B,C). The presence of strong bi-directional connections in these simulations is again indicative of a lack of synaptic competition generated by this form of STDP. When dynamic plasticity modulation is implemented, the mean weight of bi-directional connections between neurons firing synchronous, stochastic spike bursts at the peak of theta is significantly greater. Furthermore, the mean weight of asymmetric connections generated when the pre-synaptic neuron fires a complex burst and the post-synaptic neuron fires fewer spikes (i.e., when there is a negative rate correlation) are significantly lower. It is also important to note that previous research has delineated a significant correlation between the magnitude of bi-directional
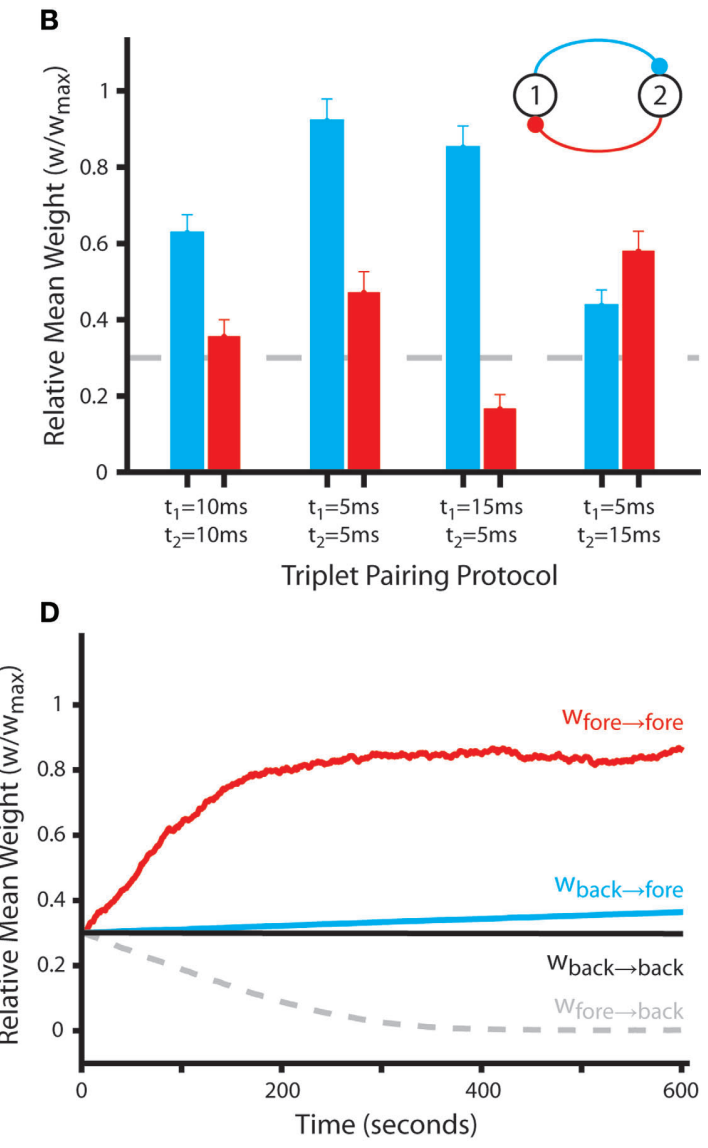

mean weight of synaptic connections between $N_{p}=490$ simulated neurons over 50 independent "rate-coded Hebbian learning" simulations, in which a subset (10\%) fire stochastically at an elevated "foreground" mean rate $\left(r_{\text {fore }} \approx 20 \mathrm{~Hz}\right)$ while the remainder fire stochastically at a lower "background" mean rate $\left(r_{\text {back }} \approx 0.1 \mathrm{~Hz}\right)$ for a period of $10 \mathrm{~s}$. Synaptic connections are grouped according to whether pre- and post-synaptic neurons fire at a foreground or background rate. Those between neurons with elevated pre- and post-synaptic firing rate are selectively and significantly potentiated, while those with solely elevated pre-synaptic firing rate are selectively and significantly depressed (Mann-Whitney $U$-test, $p<0.01$ ). The dashed line indicates the initial synaptic weight value of $w_{i j}=0.3$. (D) Synaptic weight dynamics in a typical rate-coded Hebbian learning simulation, illustrating the rapid potentiation of connections between neurons firing stochastically at an elevated rate.

connections between simultaneously active simulated neurons and the scale of recurrent axonal delays between those neurons (Bush et al., 2010b).

In summary, these results demonstrate that the phenomenological plasticity rule utilized here can approximate the primary characteristics of synaptic plasticity data obtained in vitro using either rate or temporally coded stimulation protocols. The STDP model can generate both asymmetric and bi-directional connectivity patterns, according to long-term correlations in neural activity, and thus mediate both hetero-associative and auto-associative learning in recurrently connected networks (Bush et al., 2010b). However, these emergent properties come at the cost of the inherent synaptic competition observed in previous computational models of STDP (Song et al., 2000; Song and Abbott, 2001; Bush et al., 2010a). It is 

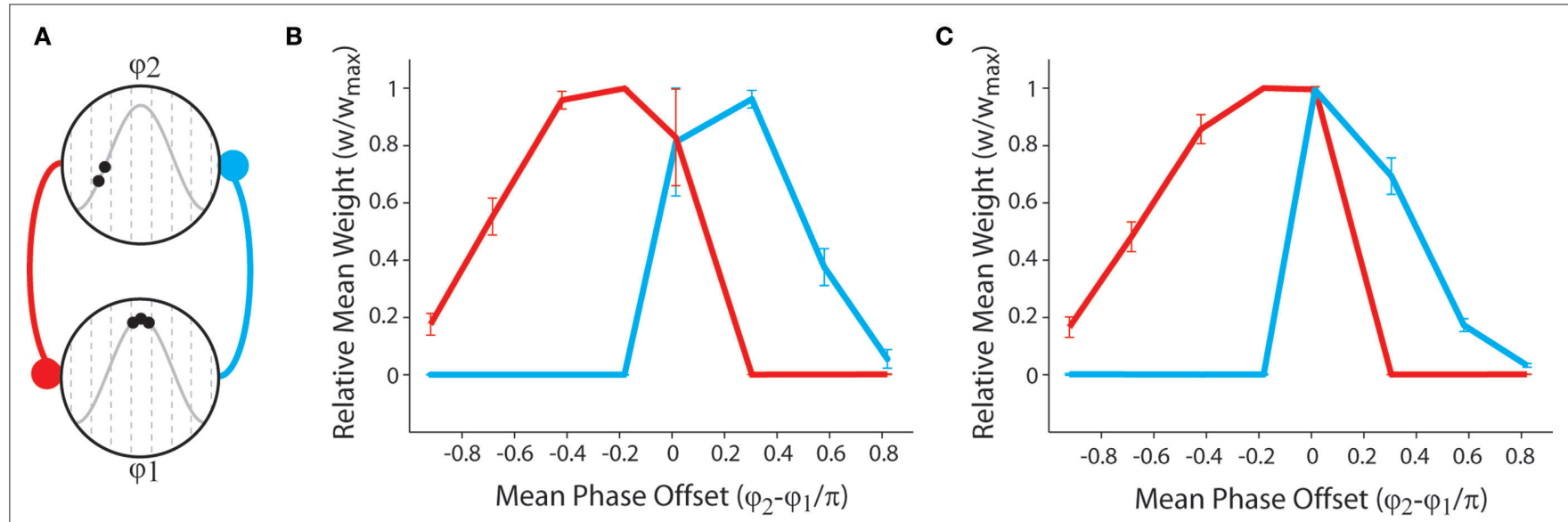

FIGURE 4 | Synaptic weight dynamics produced by the STDP rule with theta-coded spike trains that are representative of activity observed in place cells encoding for overlapping place fields within the hippocampus. (A) Illustration of neural dynamics in these simulations: in a network of $N_{P}=2$ simulated neurons, one neuron consistently fires stochastic complex spike bursts at the peak of an $8 \mathrm{~Hz}$ inhibitory theta oscillation $\left(\varphi_{1} \approx \pi\right)$, while the other fires stochastic bursts or single spikes within another theta phase window $\left(\varphi_{2^{\prime}}\right.$ see Figure 2) which differs for each set of 50 independent simulations, of length $10 \mathrm{~s}$, that are performed. (B) The mean and standard deviation of final synaptic weights for each value of $\varphi_{2}$ with no plasticity modulation. Strong asymmetric connections are generated when there is a persistent temporal correlation in neural activity (i.e., $\varphi_{2}-\varphi_{1} \neq 0$ ), while strong bi-directional connections are generated when neural activity is stochastic but approximately synchronous (i.e., $\varphi_{2}-\varphi_{1} \approx 0$ ). Furthermore, the strength of asymmetric connections is lower when pre-synaptic firing rate is higher than post-synaptic firing rate (i.e., when $\varphi_{2}-\varphi_{1}>0$ ) in accordance with the results described in Figure 3C. (C) The mean and standard deviation of final synaptic weights for each value of $\varphi_{2}$ with dynamic plasticity modulation. Note that the strength of bi-directional connections formed when there is no persistent sequence bias in pre- and post-synaptic activity (i.e., $\varphi_{1}-\varphi_{2} \approx 0$ ) is greater, and the strength of asymmetric connections generated when pre-synaptic firing rate exceeds post-synaptic firing rate (i.e., $\varphi_{2}-\varphi_{1}>0$ ) is lower when dynamic plasticity modulation is implemented. important to note that the qualitative nature of these findings do not depend explicitly upon the use of a triplet-based STDP rule (i.e., one which incorporate a $P_{++}$term), but can also be achieved by a pair-based STDP rule if certain constraints are placed on the profile of the STDP window and spike pair interactions, although the latter cannot replicate empirical data obtained with triplets of pre- and post-synaptic action potentials delivered at low frequencies (Bush et al., 2010a,b).

\section{SHUTTLE RUN SIMULATIONS}

Having established that the emergent synaptic dynamics produced by this form of STDP concur broadly with empirical data, we next implement the plasticity rule within an abstract spiking recurrent neural network model of hippocampal spatial learning. In these simulations, neural dynamics correspond hypothetically to those observed in hippocampal pyramidal neurons during spatial exploration, with theta-coded activity patterns created in simulated place cells by the phenomenological phase precession model (O'Keefe and Recce, 1993; O’Keefe, 2007; Huxter et al., 2008). We consider different navigation strategies in a square arena of equidistant place fields, each of which is encoded for by an ensemble of place cells.

Initially, neural activity corresponds hypothetically to repeated shuttle runs along a one-dimensional route of overlapping place fields. Subsequently, interconnected place cells encoding for neighboring place fields exhibit temporal correlations in firing activity with repeatedly alternating direction but approximately equal ISIs. It has been suggested that the asymmetric nature of the STDP learning window dictates that purely asymmetric connections will develop under these conditions (Skaggs et al., 1996; Wagatsuma and Yamaguchi, 2007; Song and Abbott, 2001; but see Mongillo et al.,
2005; Samura and Hattori, 2005). For the purposes of cognitive map theory, however, it is essential that strong bi-directional connections which reflect the relative location of corresponding place fields in the environment and the long-term history of navigation through this one-dimensional environment are generated (Muller et al., 1991; Burgess et al., 1994; Burgess, 2007).

Our results demonstrate that, following 10 shuttle runs along the route, the mean weight of hetero-associative connections between place cells encoding for each place field and those up to three steps in either direction on the learned route are selectively and significantly potentiated (Figures 5A,B). Strong bi-directional connections also develop between place cells that encode for the same location. When no dynamic plasticity modulation is implemented, these auto-associative connections are weaker than hetero-associative connections between place cells encoding for adjacent place fields. These results correspond closely with the synaptic dynamics observed at connections between hippocampal neurons with overlapping place fields in vivo (Figure 5G in Isaac et al., 2009).

An examination of the synaptic dynamics generated in these simulations demonstrates that, although the mean weight of hetero-associative connections remains significantly higher than that of other synaptic weights in the network, those against the direction of motion are significantly depressed during a single traversal of the route (Figures $5 \mathrm{C}, \mathbf{D}$ ). The relative magnitude of heteroassociative connections corresponding to opposing directions of traversal therefore fluctuate continually during these shuttle runs. Hence, the resultant "cognitive map" of the learned route is biased in the direction that the environment was most recently explored. This implies that, if a sequence of place fields is repeatedly traversed in a single direction, then hetero-associative connections between 

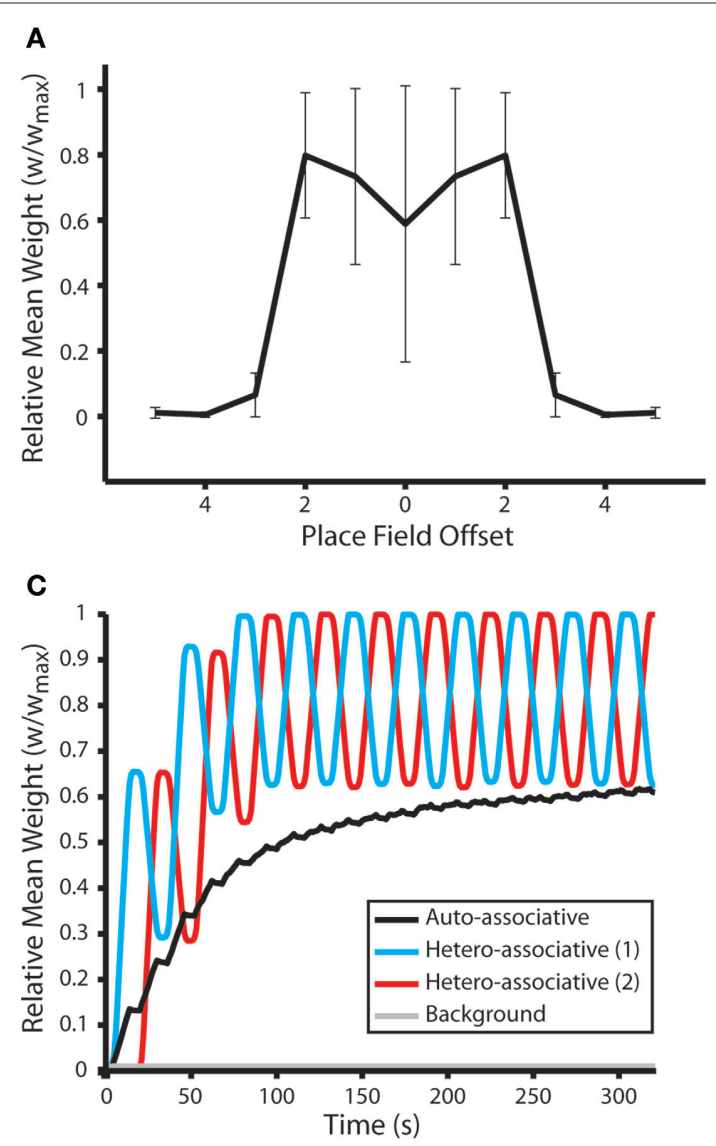

FIGURE 5 | Synaptic weight dynamics during repeated shuttle runs along a route of overlapping place fields. (A) The mean and standard deviation of final synaptic weights following 50 independent simulations of a network consisting of $N_{p}=490$ simulated neurons. Neural activity corresponds to 10 shuttle runs along a one-dimensional route of 10 place fields, each of which is encoded by the activity of 10 randomly selected place cells. Synaptic weights evolve to reflect the relative distance between corresponding place fields, in accordance with cognitive map theory, with connections between neurons that encode for the same place field or two neighboring place fields being selectively and significantly potentiated (Mann-Whitney $U$-test, $p<0.01$ ). Data illustrated here for simulations with no plasticity modulation. (B) Mean and standard deviation of final synaptic weights following 50 independent shuttle run simulations, as described for (A), when dynamic plasticity modulation is implemented. Note that synaptic
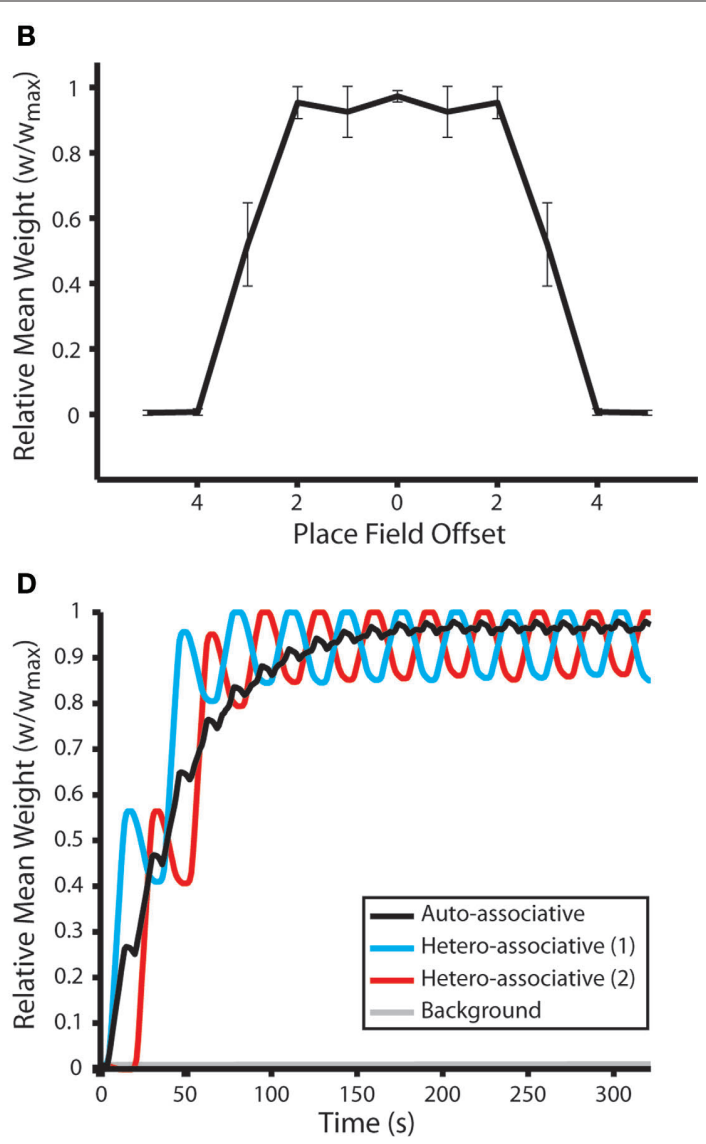

connections between a greater number of adjoining place fields are selectively and significantly potentiated in this case, and the magnitude of auto- and hetero-associative connections is also greater than that observed in the absence of plasticity modulation. (C) Synaptic dynamics during a typical shuttle run simulation in the absence of plasticity modulation. The mean weight of connections between place cells encoding for each place field on the one-dimensional route and those encoding for the same place field (auto-associative); the neighboring place fields in opposing directions along the route (hetero-associative); and all place fields that are not part of the route (background) are illustrated. Auto- and hetero-associative connections are rapidly, selectively and significantly potentiated, while the mean weight of background connections does not change significantly. (D) Synaptic dynamics during a typical shuttle run simulation in the presence of dynamic plasticity modulation, as described for (C). the corresponding place cells will become purely asymmetric, as synapses against the direction of motion undergo sustained depression (Bush et al., 2010b).

\section{RANDOM EXPLORATION SIMULATIONS}

Next, we examine the emergent synaptic dynamics produced during random exploration of a two-dimensional arena of place fields, each of which is encoded by an ensemble of place cells that exhibit theta-coded neural dynamics. Our results demonstrate that, following approximately $8 \mathrm{~min}$ of random exploration, a putative cognitive map of the environment is encoded in recurrent connections such that the relative distance between place fields for which pre- and post-synaptic place cells encode correlates with the magnitude of interconnecting synaptic weights (Figures 6A,B).
Strong, bi-directional (auto-associative) connections again develop between place cells that encode for the same location, although these are weaker than hetero-associative connections between place cells that encode for neighboring locations in the environment when no plasticity modulation is implemented. Hetero-associative connections are also more rapidly potentiated (Figure 6C), although this depends on the corresponding place fields being traversed in the necessary order, while auto-associative connections are strengthened whenever a place field is entered. An examination of sample "cognitive maps" for single place fields illustrates that uniform, omnidirectional connections between place cells are not created in all locations (Figure 6D). Hence, while the overall/mean connectivity pattern of the network is characterized by strong bi-directional connections between place cells encoding for proximate place fields 

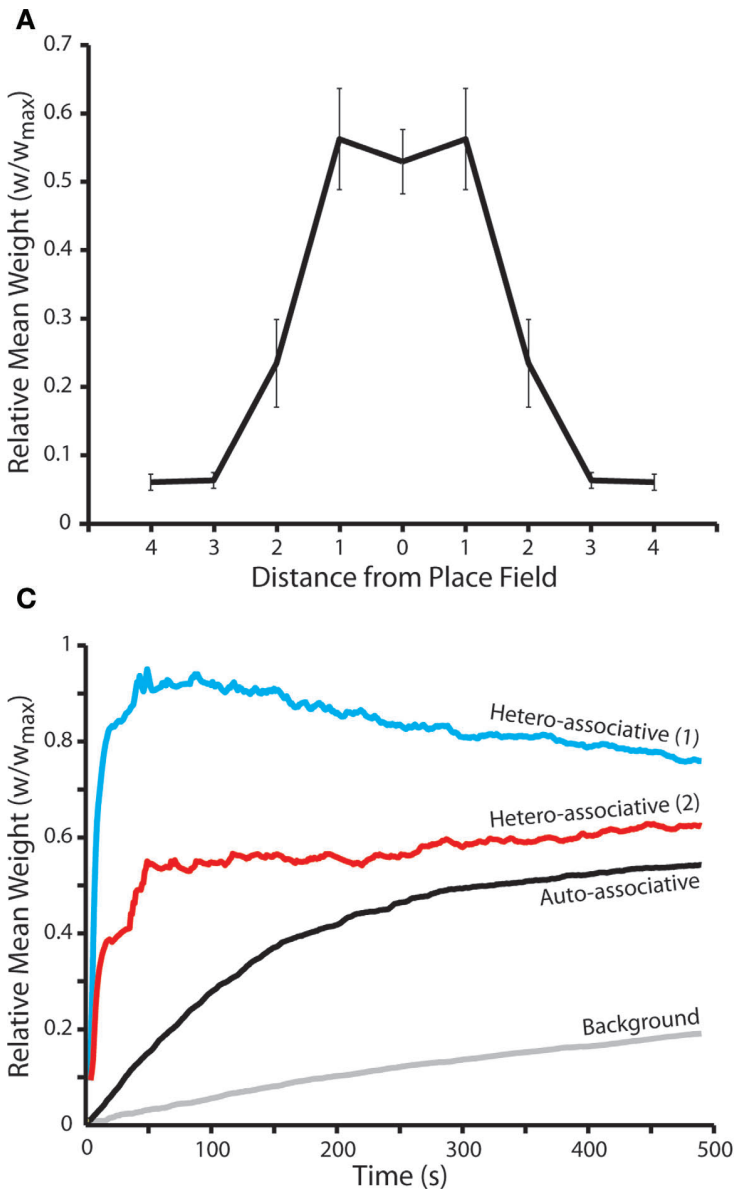

FIGURE 6 | Synaptic weight dynamics during random exploration of a square arena populated by multiple overlapping place fields. (A)The mean and standard deviation of final synaptic weights following 50 independent simulations of a network consisting of $N_{p}=490$ simulated neurons, where neural activity corresponds to the random exploration of a square environment of 49 place fields, each of which is encoded by the activity of 10 randomly selected place cells, for a total of $490 \mathrm{~s}$. Synaptic weights evolve to reflect the relative distance between corresponding place fields, in accordance with cognitive map theory, with connections between neurons that encode for the same place field, or the two neighboring place fields, being selectively and significantly potentiated (Mann-Whitney U-test, $p<0.01$ ). Data illustrated here for simulations with no plasticity modulation. (B) The mean weight of post-synaptic connections between place cells encoding for each place field and all other place cells in the network, re-arranged to correspond with the structure of the square arena. There is no significant difference between this data and that for pre-synaptic connection
B

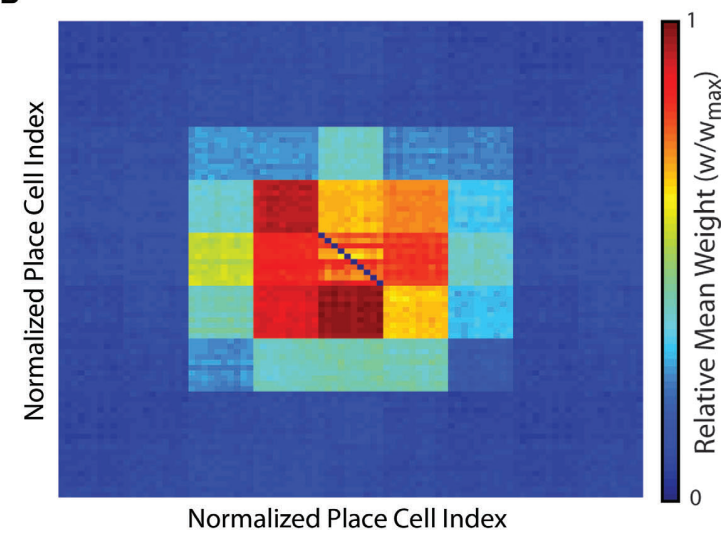

D

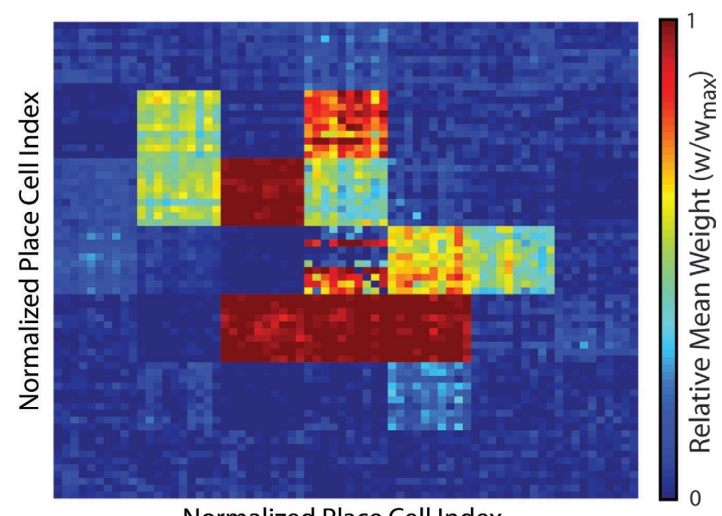

Normalized Place Cell Index weights (data not shown). (C) Synaptic dynamics during a typical random exploration simulation in the absence of plasticity modulation. The mean weight of connections between place cells encoding for each place field on the route taken and those encoding for the same place field (auto-associative); place fields that are one and two steps in any direction (hetero-associative 1 and 2

respectively); and all place fields that are not part of the route (background) are illustrated. Auto- and hetero-associative connections are rapidly, selectively and significantly potentiated, while background connections undergo modest but sustained potentiation. (D) The mean weight of post-synaptic connections between a single randomly selected place field in a typical simulation and all other place cells in the network, re-arranged to correspond with the structure of the square arena. This illustrates how the cognitive map formed for a single place field is biased by the trajectories taken through that place field during random exploration. Again, there is no significant difference between this data and that for pre-synaptic connection weights (data not shown). in the environment, local connectivity patterns tend to reflect the specific trajectories taken through that place field during random exploration. It seems likely that this effect would disappear over longer periods, as all possible trajectories through each place field are explored.

It is important to note that "background" connections in the network - i.e., synapses between place cells encoding for place fields that do not overlap - undergo modest but sustained potentiation throughout these simulations (Figure 6C). This is again indicative of the lack of synaptic competition generated by this form of STDP. Over prolonged periods of operation, the increase in background synaptic weights eventually destabilizes network operation by generating runaway recurrent excitation. The length of time for which the network maintains stable operation is inversely correlated with the background firing rate, as higher background firing rates generate a more rapid potentiation of background connections due to the increased frequency of spike pairings (data not shown). However, the inclusion of any additional mechanism that generates hetero-synaptic competition between all synaptic inputs of a single neuron can ameliorate this issue (Desai, 2004). For example, a small, global, uniform weight decay term eliminates the potentiation of background 
connections without any significant effect on the generation of auto- and hetero-associative connections between place cells encoding for overlapping place fields, and thereby stabilizes longterm network operation (data not shown).

Finally, we examine the emergent synaptic dynamics generated when neural activity corresponds to the repeated traversal of a one-dimensional route through the environment of place fields in a single direction, following the random exploration simulations described above. While the overall shape of the synaptic weight matrix changes very little (Figures 6A and 7A), the strength of connections between place cells encoding for those place fields that lie on the learned route are significantly altered. For example, Figure 7B illustrates how the cognitive map formed for a single place field (that illustrated in Figure 6D) changes when a route of seven place fields that horizontally transects that place field is subsequently traversed 10 times. Strong asymmetric connections are formed between place cells encoding for place fields that are successively activated, while those connections against the direction of motion are fully depressed.

These novel hetero-associative connections are embedded within the previously learned map, which reflects routes taken through that place field during the initial random exploration, without any significant effect on previously learned associations that do not form part of the newly learned route. Hence, the heteroassociative connections formed by place cells encoding for each place field represent the relative distance between place fields and the relative frequency with which those place fields have been sequentially traversed. These results also indicate that STDP and theta-coded neural dynamics allow rapid sequence learning, via the formation of asymmetrically connected place cell assemblies, in accordance with previous models of route learning in recurrent networks (Skaggs et al., 1996; Wagatsuma and Yamaguchi, 2007; Bush et al., 2010b).

\section{DISCUSSION}

We have presented a phenomenological STDP rule that approximates empirical synaptic plasticity data obtained using both rate and temporal stimulation protocols, and can thus generate both bidirectional and asymmetric connections in response to persistently synchronous or sequential correlations in pre- and post-synaptic activity respectively. We have subsequently demonstrated that a spiking recurrent neural network model which utilizes this STDP rule alongside theta-coded neural dynamics can mediate the development of a cognitive map during shuttle runs along a one-dimensional track or open field exploration within a two-dimensional environment, such that the geometric distance between place fields is encoded by the magnitude of bi-directional synaptic connections between place cells. This demonstrates that temporally asymmetric synaptic plasticity and phase precession are compatible with previous computational models of rate-coded cognitive map formation. Furthermore, it allows the integration of those rate-coded auto-associative network models with temporally coded, hetero-associative, spiking neural network models of route and sequence learning, and thus provides them with a firmer basis in modern neurobiology.

However, the STDP rule described here also exhibits several theoretical and functional weaknesses. Firstly, it fails to replicate recent experimental data obtained from acute hippocampal slices that suggest a requirement for multiple post-synaptic spikes to induce LTP with low frequency temporally correlated stimulation (Pike et al., 1999; Meredith et al., 2003; Wittenberg and Wang, 2006;

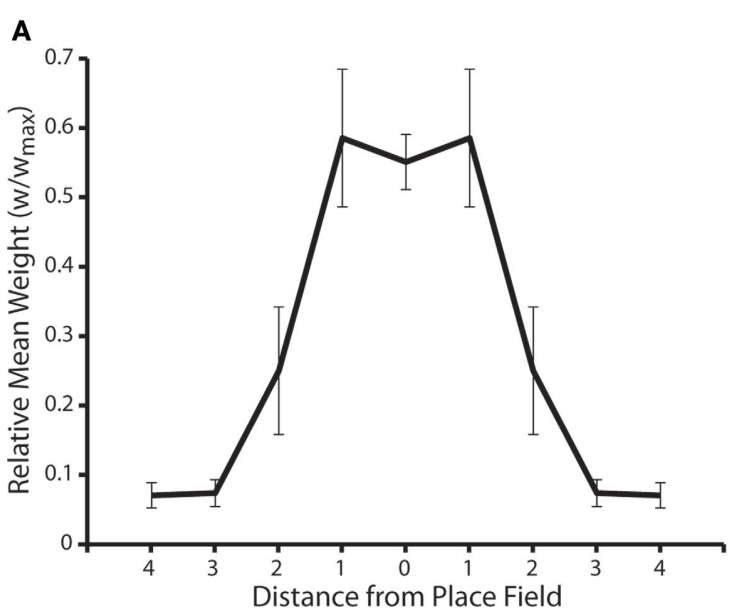

FIGURE 7 | Change in synaptic weights when a one-dimensional route is repeatedly traversed in a single direction following random exploration. (A) Mean and standard deviation of synaptic weights after an arbitrary one-dimensional route of seven place fields in length is traversed 10 times following the formation of a putative cognitive map. The only significant difference between this data and that observed prior to the traversal of the one-dimensional route (as illustrated in Figure $\mathbf{6 A}$ ) is that the standard deviation of synaptic weights between place cells encoding for a place field and those encoding for the two neighboring place fields has increased (Mann-Whitney U-test, $p<0.01$ ). (B) Changes in the cognitive map for the single randomly
B

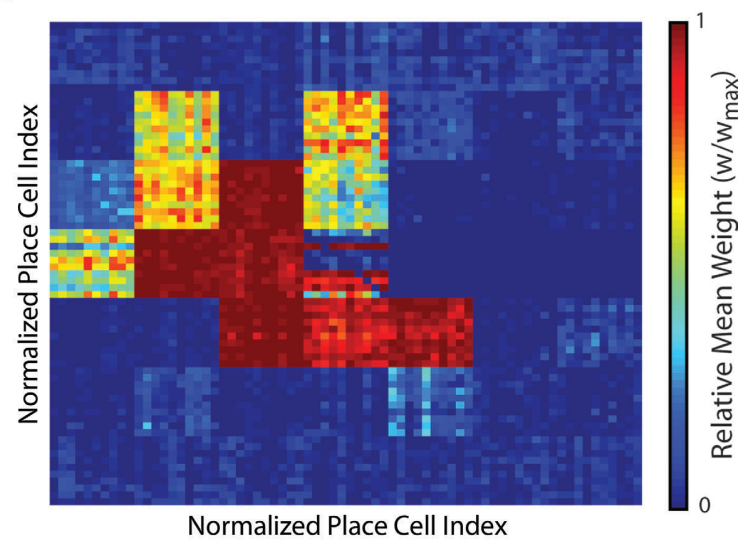

selected place field illustrated in Figure 6D following the repeated traversal of a linear route that transects the place field from right to left. Strong post-synaptic connections between place cells encoding for the central place field and those encoding for successive place fields on the route have been formed, while post-synaptic connections between place cells encoding for the central place field and those encoding for place fields against the direction of motion have been fully depressed. Conversely, the strength of connections between place cells encoding for the central place field and those encoding for all other place fields in the arena that do not form part of the newly learned route have not changed significantly (Mann-Whitney $U$-test, $p<0.01$ ). 
Buchanan and Mellor, 2007,2010), although it is important to note that empirical findings often disagree and other studies have demonstrated robust potentiation using single spike pairs in similar preparations (Kwag and Paulsen, 2009). These differences can be attributed to the multiple factors (such as membrane depolarization, the state of network inhibition, developmental stage and neuromodulatory tone) that influence post-synaptic calcium influx, which is generally recognized as the critical trigger for synaptic plasticity (Buchanan and Mellor, 2010). Furthermore, the vast majority of empirical plasticity data is obtained at the CA3-CA1 synapse, while significant differences may exist at recurrent CA3 connections. Place cells are known to receive rhythmic inhibitory input during spatial exploration, and be significantly depolarized when the corresponding place field is traversed (which concurs with the experimental procedure employed in Kwag and Paulsen, 2009), while cholinergic tone is vastly increased during stereotype learning behavior (Harvey et al., 2009; Isaac et al., 2009). Each of these factors could contribute to the induction of LTP with less intensive stimulation protocols. A wider range of experimental data might be approximated by incorporating a more explicit consideration of intra-cellular calcium dynamics, NMDA receptor kinetics and/or the backpropagation of action potentials in the dendritic tree.

Secondly, it has been empirically demonstrated that reversing the temporal order of action potentials in pairs of place cells that exhibit asymmetric spike timing cross-correlations does not prevent the induction of LTP at the interconnecting synapse, casting doubt on the existence of STDP in vivo (Isaac et al., 2009). However, it is important to note that place cells in that study exhibited only weak sequence bias in pre- and post-synaptic spike timing, while each firing at an elevated rate - circumstances that would likely generate significant bi-directional potentiation using the STDP rule presented here (Figures 3B,C). Furthermore, in the same study, the selective elimination of all spike pairings with a positive temporal correlation of $<100 \mathrm{~ms}$ prevented the induction of LTP, which suggests some requirement for temporal asymmetry to generate potentiation.

Finally, our STDP rule fails to induce any competition between the inputs of a single neuron. Some additional mechanism - such as synaptic scaling, weight decay or metaplasticity - is therefore required to ensure the long-term stability of network operation, particularly at higher background firing rates (Desai, 2004). For example, the inclusion of a slow, global weight decay term ameliorates the potentiation of background connections and thus maintains stable long-term network operation without affecting the generation of auto- and hetero-associative connections between place cells encoding for overlapping place fields (data not shown). It is important to note that this propensity for potentiation is a feature of all STDP rules that can mediate rate-coded Hebbian learning (Bush et al., 2010a,b). This might be considered a significant functional weakness and failure to replicate bi-directional changes in synaptic strength. However, it is important to note that no significant depression of synaptic connections between hippocampal place cells is observed during spatial exploration (Isaac et al., 2009). In fact, there is a convincing similarity between the data presented here and that recorded at synaptic connections between place cells whose place field have varying degrees of overlap in vivo (i.e., Figure 5A in this manuscript and Figure 5G in Isaac et al., 2009). It has also been demonstrated that synaptic connections throughout the cortex undergo net potentiation during waking, and are subsequently depressed during sleep (Vyazovskiy et al., 2008). It may be that the rapid formation of novel associations in the hippocampus is achieved at the cost of a slower but continual positive drift in background synaptic weights.

Although the neural network presented here is not intended to address the function of CA3 directly, it can also be appraised in terms of more general auto- and hetero-associative models of spatial learning. One significant omission, which therefore represents a critical direction for future research, is an examination of recall dynamics mediated by recurrent excitation, whereby external stimulation following learning generates pattern completion in encoded static or sequential activity patterns. Although we have previously demonstrated this ability following the learning of single, isolated auto- and hetero-associative activity patterns (Bush et al., 2010b), the overlap of encoded associations required by cognitive mapping generates the undirected and unrestricted spread of recurrent excitation throughout the entire network (Molter et al., 2007). Conversely, to be of any functional use, recall activity within network models of spatial learning needs to be biased by goal-directed excitatory or inhibitory input (Burgess, 2007). It seems feasible that inhibitory plasticity during spatial learning could contribute to disambiguating overlapping encoded sequences during subsequent reactivation via putative winnertake-all dynamics. This highlights a need for the inclusion of feedback inhibition in future extensions of this model, which might also dynamically regulate the firing rate of background neurons, reducing the potentiation of redundant synaptic connections and thus contributing to the long-term stability of network operation.

Finally, it is unclear to what extent rate-coded models of cognitive map formation can explain the involvement of the hippocampus in spatial learning and navigation. There is no evidence for fixed point attractor dynamics in CA3, and it remains to be seen if models of cognitive mapping can be reconciled with the transient, temporally coded cell assembly dynamics that characterize hippocampal activity during exploration (Harris, 2005). Furthermore, it is generally accepted that the role of the hippocampus in declarative memory is time-limited, and recent experimental evidence demonstrates that the neocortex is involved in spatial learning and memory consolidation from a very early stage (Leon et al., 2010). In accordance with standard "two stage" models of declarative memory processing, it seems likely that a global cognitive map would ultimately be stored in the neocortex, while recent routes or environments - "episodes" encoded in a single trial - are stored for a limited period in the hippocampus for rapid integration with this "semantic" spatial knowledge (McClelland et al., 1995). The exact contribution of the hippocampus and neocortex to spatial memory and goal-directed navigation remains to be elucidated.

\section{ACKNOWLEDGMENTS}

The authors would like to thank Jesper Sjostrom, Bart Baddeley, the anonymous referees and members of the CCNR for useful discussions during the preparation of this manuscript. This work was supported by Wellcome VIP funding and the EPSRC grant EP/H024638/1. 


\section{REFERENCES}

Baker, J. L., and Olds, J. L. (2007). Theta phase precession emerges from a hybrid computational model of a CA3 place cell. Cogn. Neurodyn. 1, 237-248.

Bi, G. Q., and Poo, M. M. (1998). Synaptic modifications in cultured hippocampal neurons: dependence on spike timing, synaptic strength, and postsynaptic cell type. J. Neurosci. 18, 10464-10472.

Bienenstock, E. L., Cooper, L. N., and Munro, P. W. (1982). Theory for the development of neuron selectivity: orientation specificity and binocular interaction in visual cortex. J. Neurosci. 2, 32-48.

Bliss, T. V., and Lomo, T. (1973). Longlasting potentiation of synaptic transmission in the dentate area of the anaesthetized rabbit following stimulation of the perforant path. J. Physiol. 232, 331-356.

Buchanan, K. A., and Mellor, J. R. (2007). The development of synaptic plasticity induction rules and the requirement for postsynaptic spikes in rat hippocampal CA1 pyramidal neurones. J. Physiol. 585, 429-445.

Buchanan, K. A., and Mellor, J. R. (2010). The activity requirements for spike timing-dependent plasticity in the hippocampus. Front. Syn. Neurosci. 2:11. doi: 10.3389/ fnsyn.2010.00011.

Burgess, N. (2007). "Computational models of the spatial and mnemonic functions of the hippocampus," in The Hippocampus Book, eds P. Andersen, R. Morris, D. Amaral, T. Bliss, and J. O'Keefe (Oxford: Oxford University Press), 715-749.

Burgess, N., Recce, M., and O’Keefe, J. (1994). A model of hippocampal function. Neural Netw. 7, 1065-1081.

Bush, D., Philippides, A., Husbands, P., and O'Shea, M. (2010a). Reconciling the STDP and BCM models of synaptic plasticity in a spiking recurrent neural network. Neural Comput. 22, $1-27$.

Bush, D., Philippides, A., Husbands, P., and O'Shea, M. (2010b). Dual coding with STDP in a spiking recurrent neural network model of the hippocampus. PLoS Comput. Biol. 6, e1000839. doi: 10.1371/journal. pcbi. 1000839 .

Buzsaki, G. (2006). Rhythms of the Brain. Oxford: Oxford University Press.

de Almeida, L., Idiart, M., and Lisman, J. E. (2007). Memory retrieval time and memory capacity of the CA3 network: role of gamma frequency oscillations. Learn. Mem. 14, 795-806.

Debanne, D., Gahwiler, B. H., and Thompson, S. M. (1998). Long-term synaptic plasticity between pairs of individual CA3 pyramidal cells in rat hippocampal slice cultures. J. Physiol. 507, 237-247.

Desai, N. S. (2004). Homeostatic plasticity in the CNS: synaptic and intrinsic forms. J. Physiol. (Paris) 97, 391-402.

Dudek, S. M., and Bear, M. F. (1992). Homosynaptic long-term depression in area CA1 of hippocampus and effects of N-methyl-D-aspartate receptor blockade. Proc. Natl. Acad. Sci. U.S.A. 89, 4363-4367.

Frerking, M., Schulte, J., Wiebe, S. P., and Stäubli, U. (2005). Spike timing in CA3 pyramidal cells during behavior: implications for synaptic transmission. J. Neurophysiol. 94, 1528-1540.

Harris, K. D. (2005). Neural signatures of cell assembly organization. Nat. Rev. Neurosci. 6, 399-407.

Harvey, C.D., Collman, F., Dombeck, D.A. and Tank, D. W. (2009). Intracellular dynamics of hippocampal place cells during virtual navigation. Nature 461, 941-946.

Hebb, D. O. (1949). Organisation of Behaviour. New York: Wiley.

Hopfield, J. J. (1982). Neural networks and physical systems with emergent collective computational abilities. Proc. Natl. Acad. Sci. U.S.A. 79, 2554-2558.

Huxter, J. R., Senior, T. J., Allen, K., and Csicsvari, J. (2008). Theta phasespecific codes for two-dimensional position, trajectory and heading in the hippocampus. Nat. Neurosci. 11, 587-594.

Hyman, J. M., Wyble, B. P., Goyal, V., Rossi, C. A., and Hasselmo, M. E. (2003). Stimulation in hippocampal region CAl in behaving rats yields LTP when delivered to the peak of theta and LTD when delivered to the trough. J. Neurosci. 23, 11725-11731.

Isaac, J.T.R., Buchanan, K.A., Muller, R. U., and Mellor, J.R. (2009). Hippocampal place cell firing patterns can induce long-term synaptic plasticity in vitro. J. Neurosci. 29, 6840-6850.

Izhikevich, E. M. (2004). Which model to use for cortical spiking neurons? IEEE Trans. Neural Netw. 15, 1063-1070.

Jensen, O., and Lisman, J.E. (1996). Novel lists of $7 \pm 2$ known items can be reliably stored in an oscillatory shortterm memory network: interaction with long-term memory Learn. Mem. 3, 257-263.

Kwag, J., and Paulsen, O. (2009). The timing of external input controls the sign of plasticity at local synapses. Nat. Neurosci. 12, 1219-1221.
Leon, W.C., Bruno, M.A., Allard, S., Nader K., and Cuello,A.C.(2010).Engagement of the PFC in consolidation and recall of recent spatial memory. Learn. Mem. 17, 297-305

Malenka, R. C., and Bear, M. F. (2004). LTP and LTD: an embarrassment of riches. Neuron 44, 5-21.

Marr,D. (1971). Simple memory: a theory for archicortex. Philos. Trans. R. Soc. Lond., B, Biol. Sci. 262, 23-81.

Martin, S. J., Greenwood, P.D., and Morris, R. G. (2000). Synaptic plasticity and memory: an evaluation of the hypothesis. Annu. Rev. Neurosci. 23, 649-711.

Maurer, A. P., and McNaughton, B. L. (2007). Network and intrinsic cellular mechanisms underlying theta phase precession of hippocampal neurons. Trends Neurosci. 7, 325-333.

McClelland, J. L., McNaughton, B. L., and O'Reilly, R. C. (1995). Why there are complementary learning systems in the hippocampus and cortex: insights from the successes and failures of connectionist models of learning and memory. Psychol. Rev. 102, 419-457.

McNaughton, B. L., Battaglia, F. P., Jensen, O., Moser, E. I., and Moser, M.-B. (2006). Path integration and the neural basis of the 'cognitive map'. Nat. Rev. Neurosci. 7, 663-678.

Meredith, R. M., Floyer-Lea, A. M., and Paulsen, O. (2003). Maturation of long-term potentiation induction rules in rodent hippocampus: role of GABAergic inhibition. J. Neurosci. 23, 11142-11146.

Miles, R. (1990). Synaptic excitation of inhibitory cells by single CA3 pyramidal cells of the guinea pig in vitro. $J$. Physiol. 428, 61-77.

Molter, C., Sato, N., and Yamaguchi, Y. (2007). Reactivation of behavioural activity during sharp waves: a computational model for two stage hippocampal dynamics. Hippocampus 17, 201-209.

Mongillo, G., Curti, E., Romani, S., and Amit, D. J. (2005). Learning in realistic networks of spiking neurons and spike-driven plastic synapses. Eur. J. Neurosci. 21, 3143-3160.

Morris, R. (2007). "Theories of hippocampal function," in The Hippocampus Book, eds P. Andersen, R. Morris, D. Amaral, T. Bliss, and J. O'Keefe (Oxford: Oxford University Press), 581-713.

Morris, R. G., Garrud, P., Rawlins, J. N., and O'Keefe, J. (1982). Place navigation impaired in rats with hippocampal lesions. Nature 297, 681-683.

Muller, R. U., Kubie, J. L., and Saypoff, R. (1991). The hippocampus as a cognitive graph (abridged version). Hippocampus 1, 243-246.
Neves, G., Cooke, S. F., and Bliss, T. V. P. (2008). Synaptic plasticity, memory and the hippocampus: a neural network approach to causality. Nat. Rev. Neurosci. 9, 65-75.

O’Keefe, J. (2007). "Hippocampal neurophysiology in the behaving animal," in The Hippocampus Book, eds P. Andersen, R. Morris, D. Amaral, T. Bliss, and J. O'Keefe (Oxford: Oxford University Press), 475-548.

O’Keefe, J., and Dostrovsky, J. (1971). The hippocampus as a spatial map. Preliminary evidence from unit activity in the freely-moving rat. Brain Res. 34, 171-175.

O'Keefe, J., and Nadel, L. (1978). The Hippocampus as a Cognitive Map. Oxford: Oxford University Press.

O'Keefe, J., and Recce, M. L. (1993). Phase relationship between hippocampal place units and the EEG theta rhythm. Hippocampus 3, 317-330.

Pfister, J.-P., and Gerstner, W. (2006). Triplets of spikes in a model of spike timing-dependent plasticity. $J$. Neurosci. 26, 9673-9682.

Pike, F. G., Meredith, R. M., Olding, A. W. A., and Paulsen, O. (1999). Postsynaptic bursting is essential for 'Hebbian' induction of associative long-term potentiation at excitatory synapses in rat hippocampus. $J$. Physiol. 518, 571-576.

Ranck, J. B. (1973). Studies on single neurons in dorsal hippocampal formation and septum in unrestrained rats. I: Behavioural correlates and firing repertoires. Exp. Neurol. 41, 461-531.

Rolls, E. T. (2007). An attractor network in the hippocampus: theory and neurophysiology. Learn. Mem. 14, 714-731.

Rolls, E. T. (2008). Memory, Attention and Decision Making. Oxford: Oxford University Press.

Rolls, E. T., and Kesner, R. P. (2006). A computational theory of hippocampal function, and empirical tests of the theory. Prog. Neurobiol. 79, 1-48.

Samura, T., and Hattori, M. (2005). Hippocampal memory modification induced by pattern completion and spike-timing dependent synaptic plasticity. Int. J. Neural Syst. 15, 13-22.

Skaggs, W. E., McNaughton, B. L., Wilson, M. A., and Barnes, C. A. (1996). Theta phase precession in hippocampal neuronal populations and the compression of temporal sequences. Hippocampus 6, 149-172.

Song, S., and Abbott, L. F. (2001). Cortical development and remapping through spike timing-dependent plasticity. Neuron 32, 339-350.

Song, S., Miller, K. D., and Abbott, L. F (2000). Competitive Hebbian learn- 
ing through spike-timing-dependent synaptic plasticity. Nat. Neurosci. 3, 919-926.

Vyazovskiy, V. V., Cirelli, C., PfisterGenskow, M., Faraguna, U., and Tononi, G. (2008). Molecular and electrophysiological evidence for net synaptic potentiation in wake and depression in sleep. Nat. Neurosci. 11, 200-208.

Wagatsuma, H., and Yamaguchi, Y. (2007). Neural dynamics of the cognitive map in the hippocampus. Cogn. Neurodyn. 1, 119-141.

Wang, H. X., Gerkin, R. C., Nauen, D. W., and Bi, G.-Q. (2005). Coactivation and timing-dependent integration of synaptic potentiation and depression. Nat. Neurosci. 8, 187-193.

Wittenberg, G. M., and Wang, S.-S. H. (2006). Malleability of spike-timing-dependent plasticity at the CA3-CA1 synapse. J. Neurosci. 26, 6610-6617.
Conflict of Interest Statement: The authors declare that the research was conducted in the absence of any commercial or financial relationships that could be construed as a potential conflict of interest.

Received: 17 February 2010; accepted: 24 September 2010; published: 15 October 2010.

Citation: Bush D, Philippides A, Husbands $P$ and O'Shea $M$ (2010) Spike-timing dependent plasticity and the cognitive map. Front. Comput. Neurosci. 4:142. doi: 10.3389/fncom.2010.00142

Copyright (C) 2010 Bush, Philippides, Husbands and O'Shea. This is an openaccess article subject to an exclusive license agreement between the authors and the Frontiers Research Foundation, which permits unrestricted use, distribution, and reproduction in any medium, provided the original authors and source are credited. 